\title{
Effectiveness of Cognitive-Behavioral Group Therapy on Coping Styles in Multiple Sclerosis Patients
}

\section{ARTICLE INFO}

\section{Article Type}

Original Research

\section{Authors}

Sadri Damirchi E.* $P h D$,

Agazadehasl M. ${ }^{1} M A$

\begin{abstract}
A B S T RACT
Aims Stress is one of the most important factors in aggravation and relapse of the symptoms of multiple sclerosis (MS). The cognitive-behavioral approach is one of the most effective methods to cope with and prevent stress, as well as self-control, in patients with MS. The aim of the study was to determine the effectiveness of cognitive-behavioral group therapy on the coping styles of the patients with MS.

Materials \& Methods In the controlled pretest-posttest quasi-experimental study, 80 patients with MS, who were members of Urmia MS Society, were studied in 2015. The subjects, selected via simple random sampling method, were randomly divided into two 40-person groups including experimental and control groups. Data was collected using the coping style questionnaire. Eight cognitive-behavioral group therapy sessions were conducted in experimental group, while control group received no intervention. Data was analyzed by SPSS 20 software using one-way ANOVA.

Findings In experimental group, the cognitive-behavioral group therapy training significantly increased the mean problem-focused coping style score $(\mathrm{p}=0.04)$, while significantly reduced the mean scores of emotion-focused $(\mathrm{p}=0.05)$ and avoidance $(\mathrm{p}=0.02)$ coping styles compared to control group.

Conclusion The intervention based on the cognitive-behavioral group therapy increases the problem-focused coping styles, while reduces the emotion-focused and avoidance coping styles in the patients with MS.
\end{abstract}

Keywords Coping Skills; Therapy, Cognitive Behavioral; Multiple Sclerosis

\section{I T A T I O N L IN KS}

[1] Efficacy of group therapy on reduction of psychological signs of multiple sclerosis patients [2] The role of stress-response systems for the pathogenesis and progression of MS [3] Psychosocial interventions in people with multiple sclerosis [4] Association between stressful life events and exacerbations in multiple sclerosis: A meta-analysis [5] Psychological stress and the human immune system: A meta-analytic study of 30 years of inquiry [6] Chlamydia pneumoniae and the risk for exacerbation in multiple sclerosis patients [7] Social Support and Resilience to Stress [8] Antecedents of coping with the disease in patients with multiple sclerosis: A qualitative content analysis [9] Comparison between styles of coping with stress in patient withmultiple sclerosis and healthy people in the east of Mazandaran [10] Effects of stress management training based on cognitive-behavioral therapy on chronic fatigue and coping strategies in multiple sclerosis patients [11] Rosenberger J, Szilasiova J, et al. Coping and its importance for quality of life in patients with multiple sclerosis [12] Characteristics of coping strategies in patients with multiple sclerosis [13] The relationship between mental health and coping strategies in citizenship of Khoramabad City [14] Multidimensional assessment of coping: A critical evaluation [15] The relationship between diffuse axonal damage and fatigue in multiple sclerosis [16] Impact of cognitive impairment on coping strategies in multiple sclerosis [17] Health Status of people with multiple sclerosis a community mail survey [18] The effect of cognitive -behavioral group training method on the stress in patients with multiple sclerosis [19] Psychological interventions for multiple sclerosis [20] Personality trait and coping strategies in multiple sclerosis: Neuropsychological and radiological correlation [21] Sample size in clinical and experimental trials [22] The effect of cognitive-behavioral group therapy on depressive symptoms in people with type 2 diabetes: A randomized controlled clinical trial [23] A replicated prospective investigation of life stress, coping, and depressive symptoms in multiple sclerosis

\section{Article History}

Received: April 20, 2016

Accepted: January 21, 2017

ePublished: March 25, 2017 
مولتييلاسكلروزيس است. همجنين استرس بهاعنوان عارضهاى از از

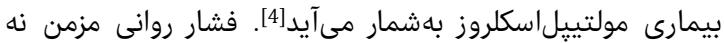

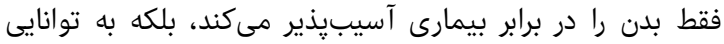

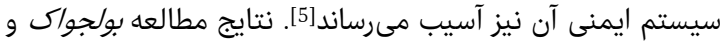

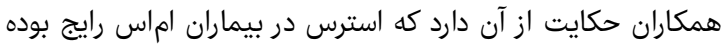

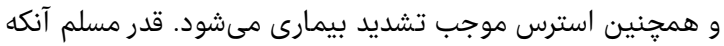

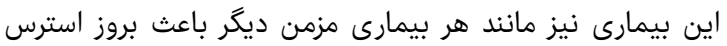

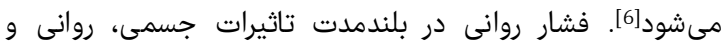

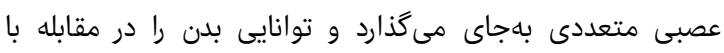

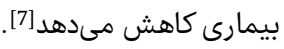

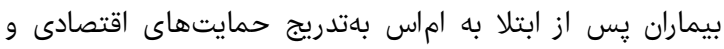

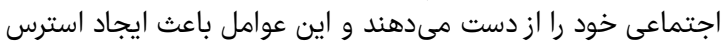

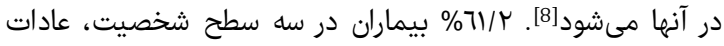

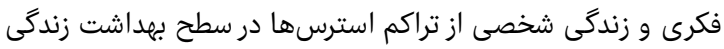

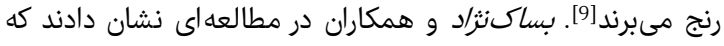

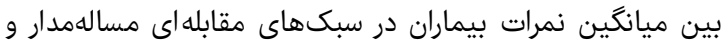

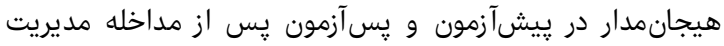

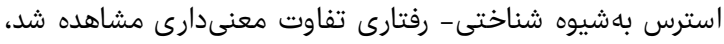

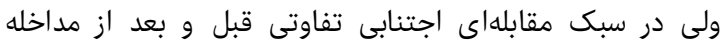

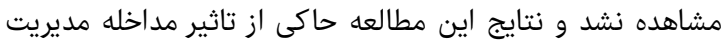

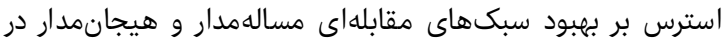

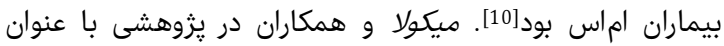

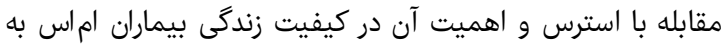

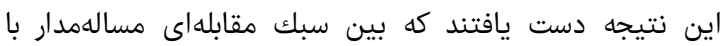

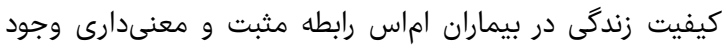

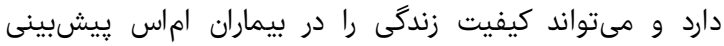

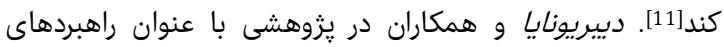

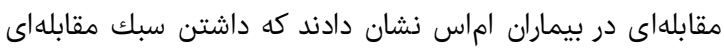

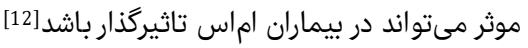

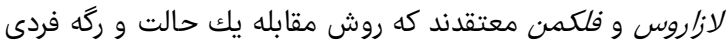

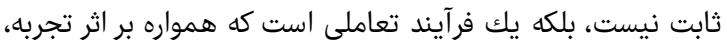

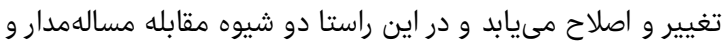

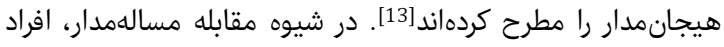

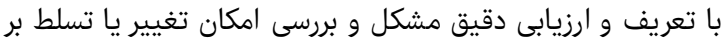

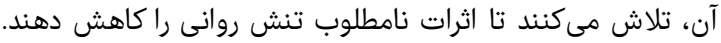

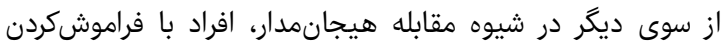

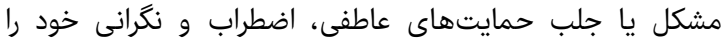

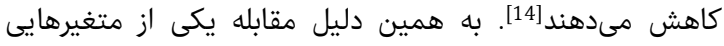

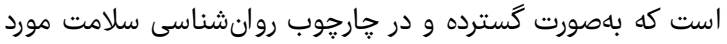

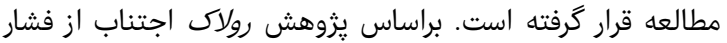

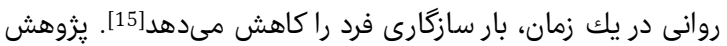

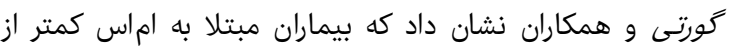

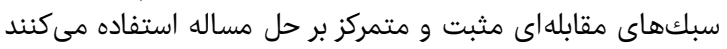

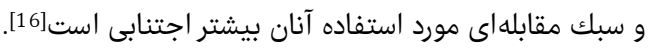

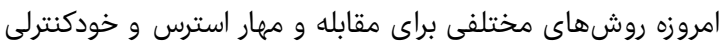

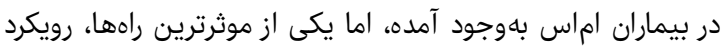

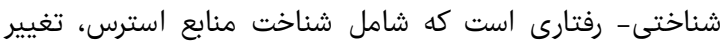

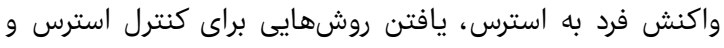

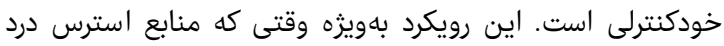

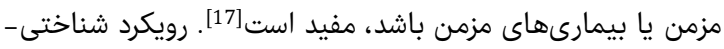

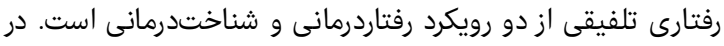

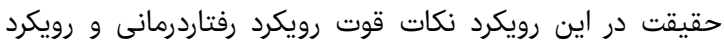
دوره سr، شماره r، بهار كوسا

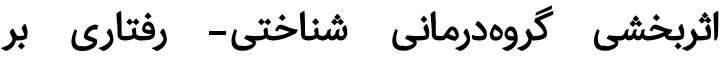

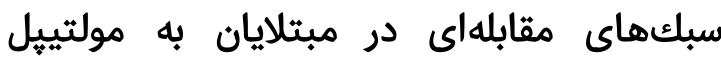 اسكلروزيس}

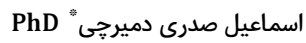
كرّه علوم تربيتى، دانشكده علوم تربيتى و روان شناسى، دانشكاه محقق اردبيلى، إنى

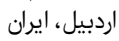

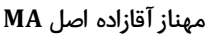

دانشكده علوم انسانى، واحد اروميه، دانشكاه آزاد اسلامى، اروميه، ايران

קكيده

اهداف: استرس يكى از عوامل مهم در تشديد و و عود علايم بيمارى

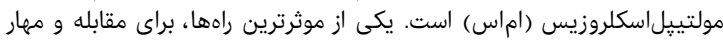

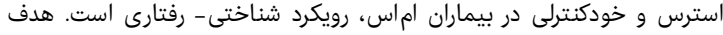

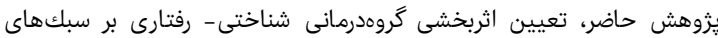

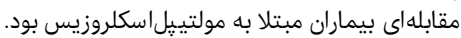

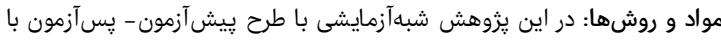

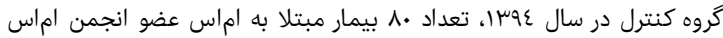

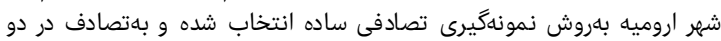

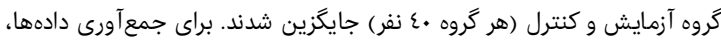

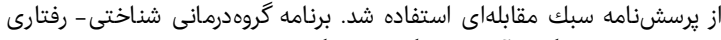

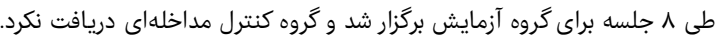

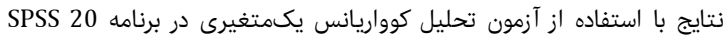

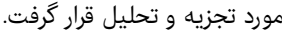

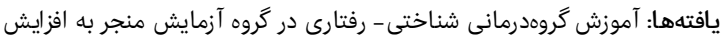

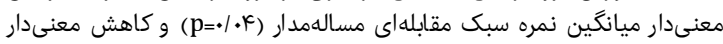

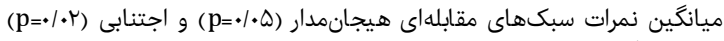

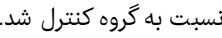

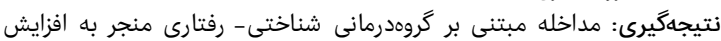

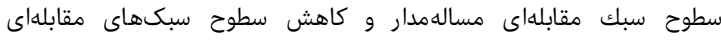

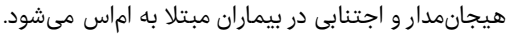
كليدواثَها: راهبردهاى مقابلهاى، درمان شناختى ـ رفتارى، مولتيبيل اسكلروزيس

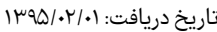

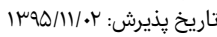

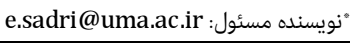

مقدمه

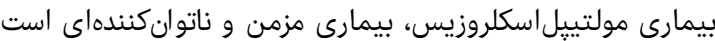

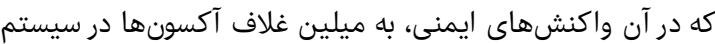

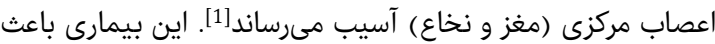

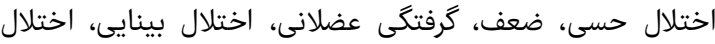

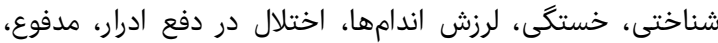

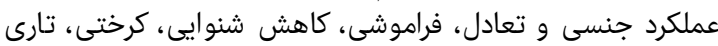

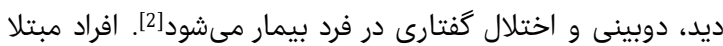

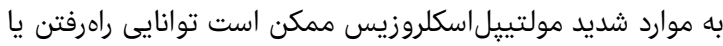

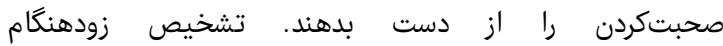

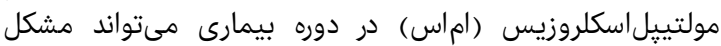

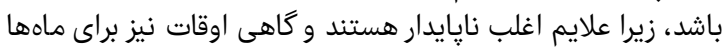

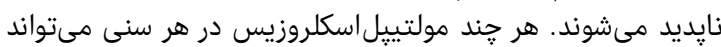

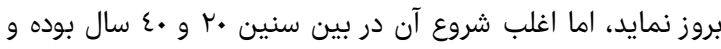

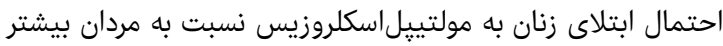

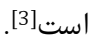

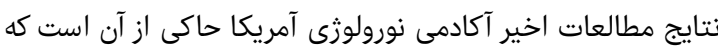

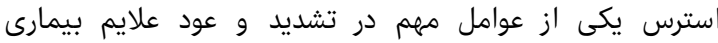

فصل نامه علمى - يُروهشى افق دانش 


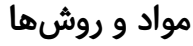

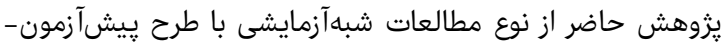

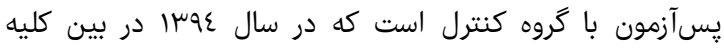

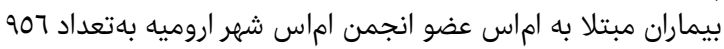

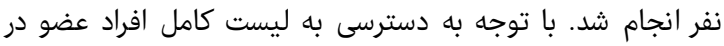

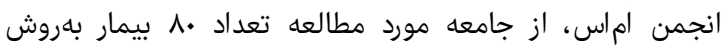

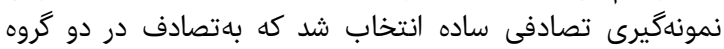

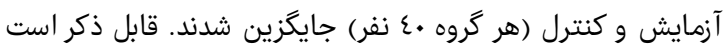

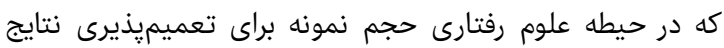

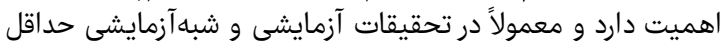

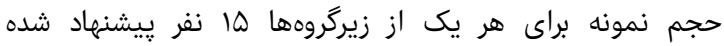

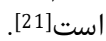

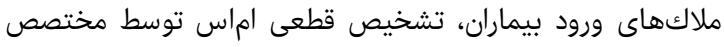

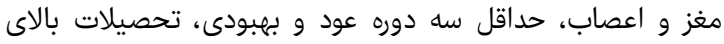

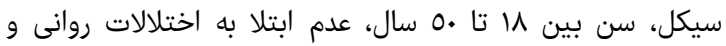

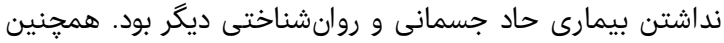

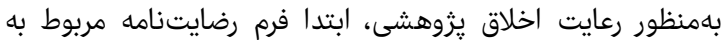

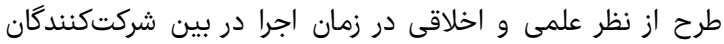

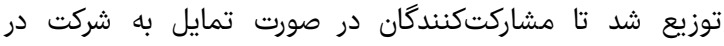

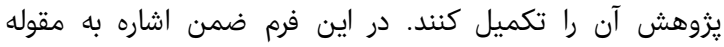

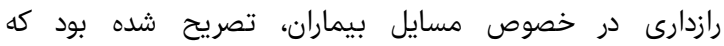

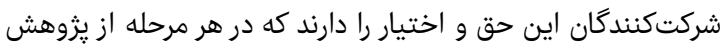

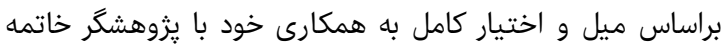

دهند.

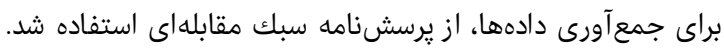

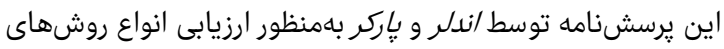

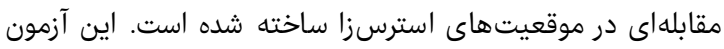

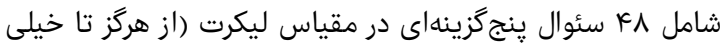

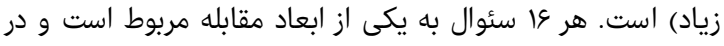

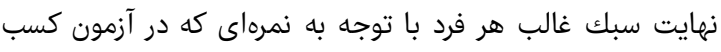

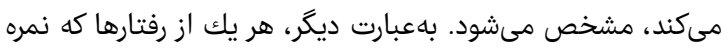

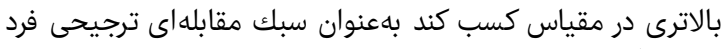

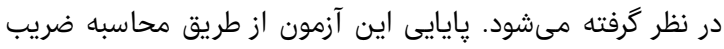

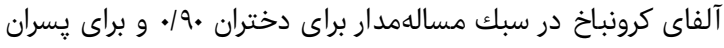

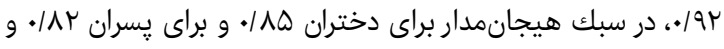

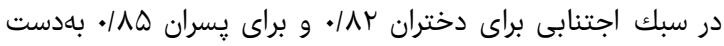

آمد [14].].

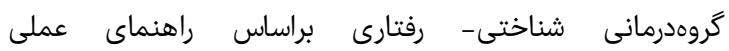

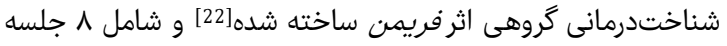

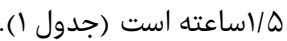

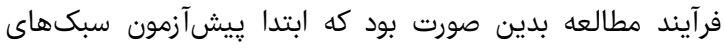

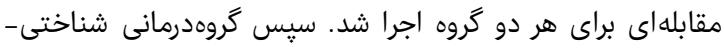

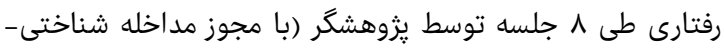

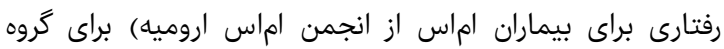

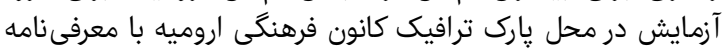

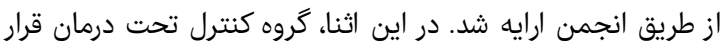

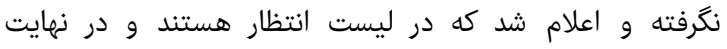

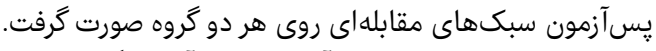

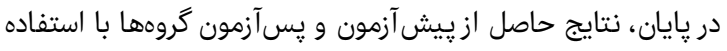

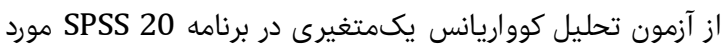

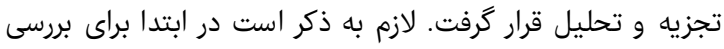

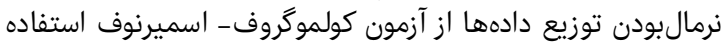

شناختدرمانى يعنى عينى

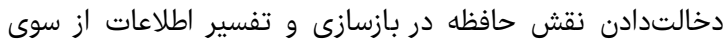

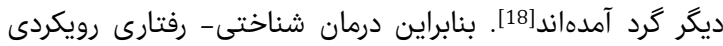

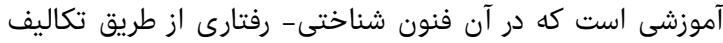

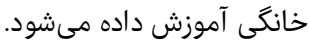

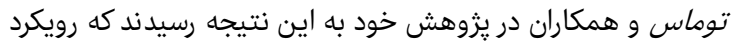

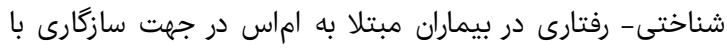

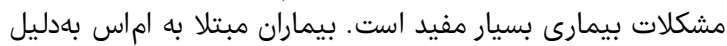

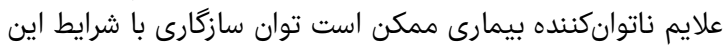

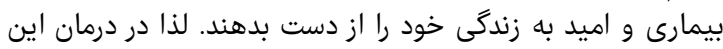

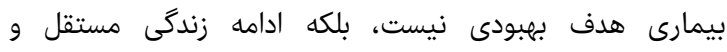

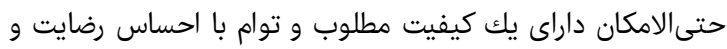

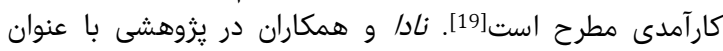

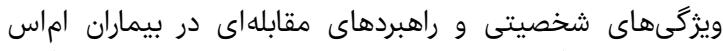

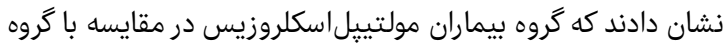

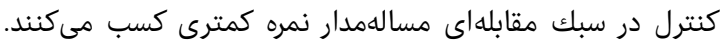

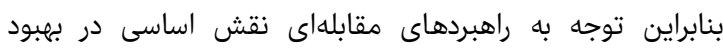

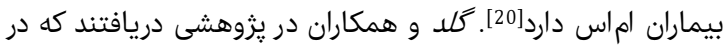

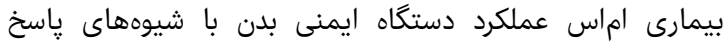

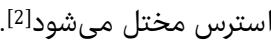

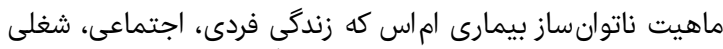

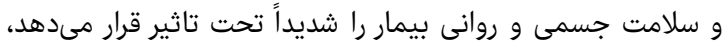

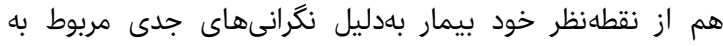

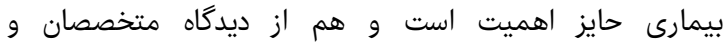

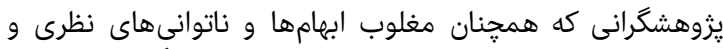

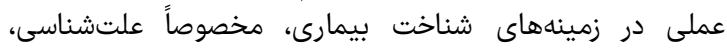

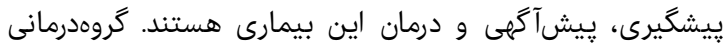

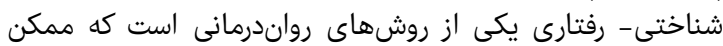

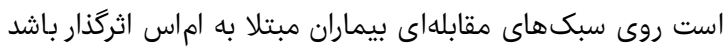

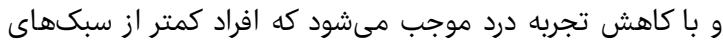

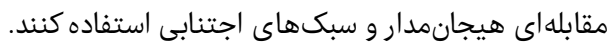

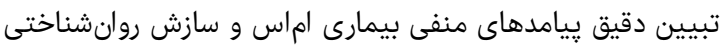

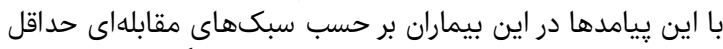

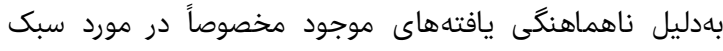

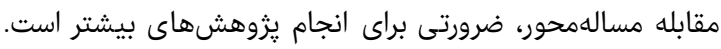

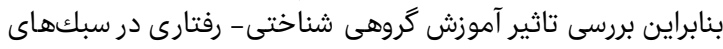

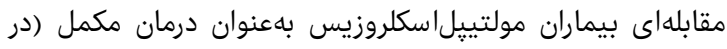

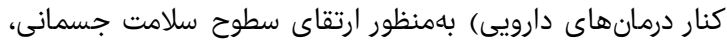

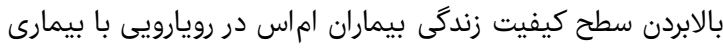

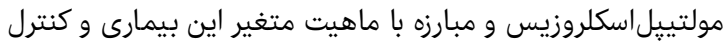

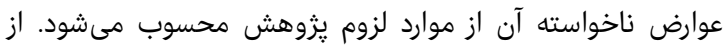

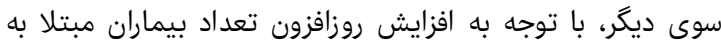

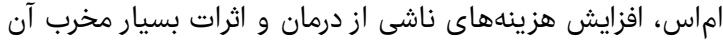

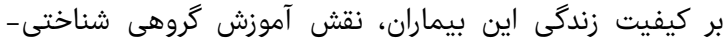

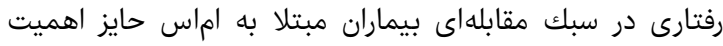

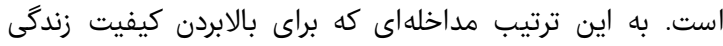

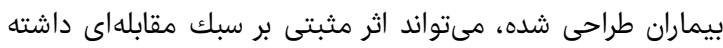

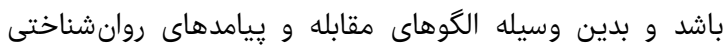

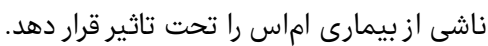

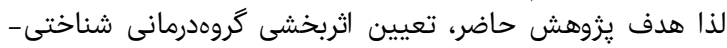

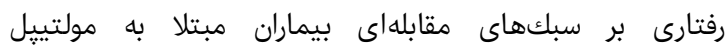

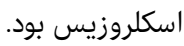


• rا اسماعيل صدرى دميرجى و مهناز آقازاده اصل

هدف يزوهش حاضر، بررسى اثربخشى گروهدرمانى شناختى-

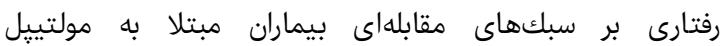

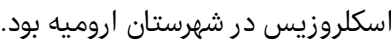

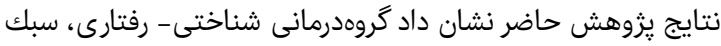

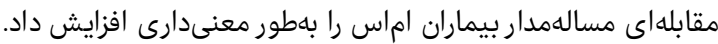

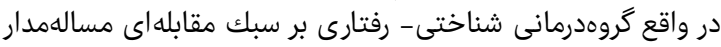

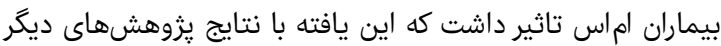

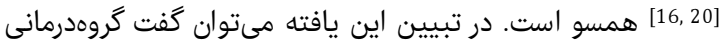

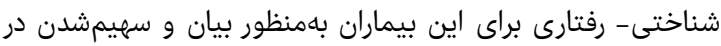

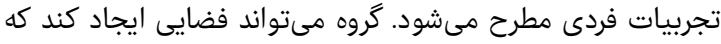

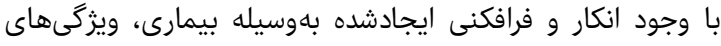

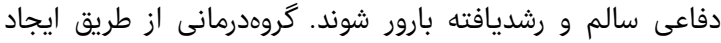

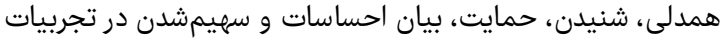

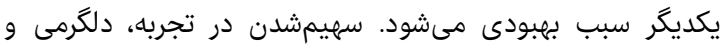

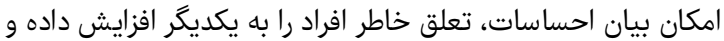

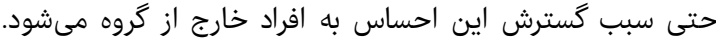

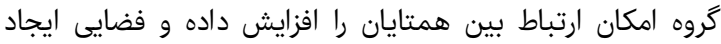

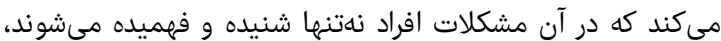

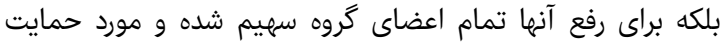

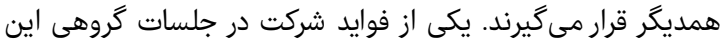

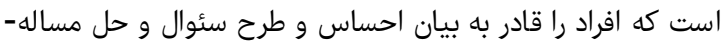

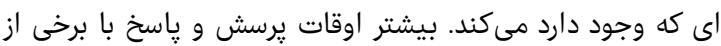

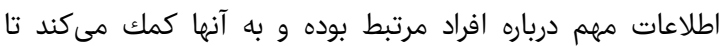

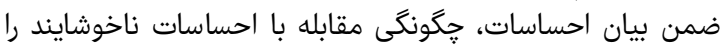

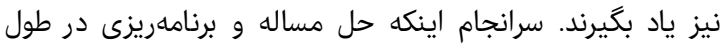

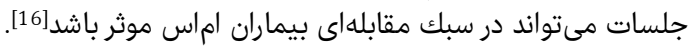

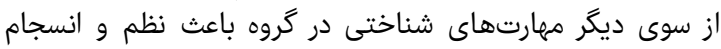

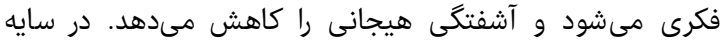

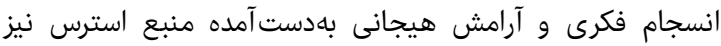

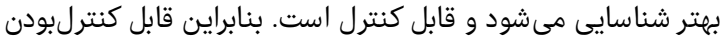

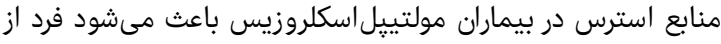

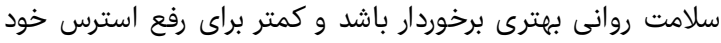

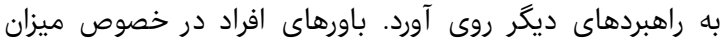

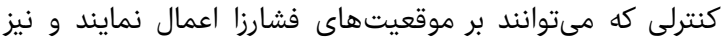

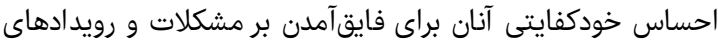

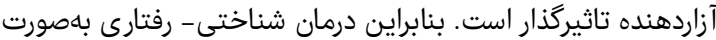

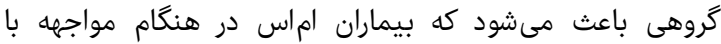

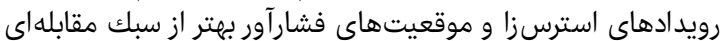

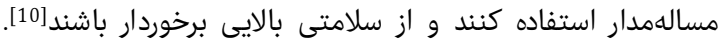

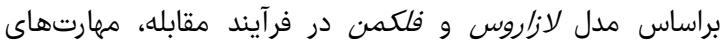

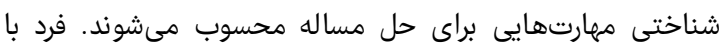

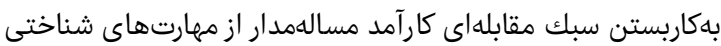

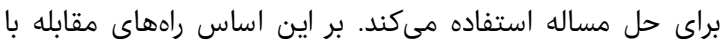

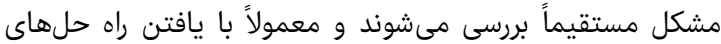

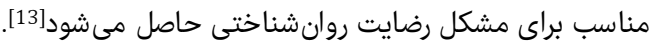

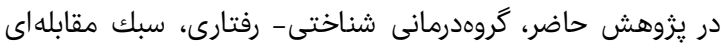

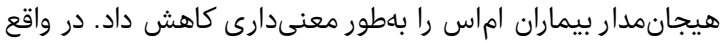

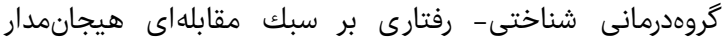

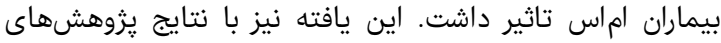

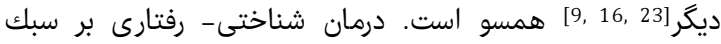

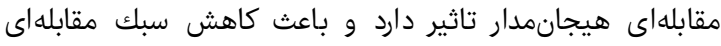

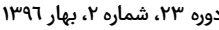

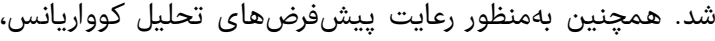

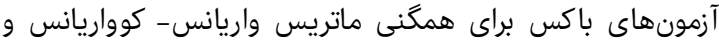

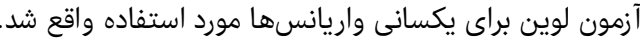

جدول () محتواى جلسات گ گرودرمانى شناختى ـ رفتارى جلسه اول معارفه، آشنايى با قوانين و اهداف گروه، بيان هدف جلسه، آشنايى با فرآيند A

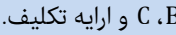

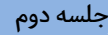

مرور تكاليف، آكاهى اعضا از ابعاد اصلى نظريههاى شنائ شاختى هيجان، آشنايى اعضا با تحريف هاى شناختى يا خطاهاى منطقى و اعضى ارايه تكليف إدايف

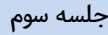
مرور تكاليف و مطالب جلسه قبل، آشنايى اعضاى گروه با مثلث شناختى منفى و خطاى منطقى و ارايه تكليف. جلسه جهارم

مرور تكاليف جلسه قبل، آموزش به اعضا درباره ماهيت طرحوارهها و ارتبار ارتباط بين

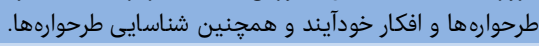
جلسه ينجم مرور تكاليف جلسه قبل، آشنايى با ييكان عمودى و انواع باورها و ارايه تكليف. جلسه ششم تكرو تكاليف

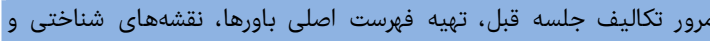

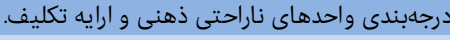
جلسه هفتم

مرور تكاليف جلسه قبل، تغييريذيرى باورها، تحليل عينى و تحليل همسانى باورها. جلسه هشتم مرور مطالب جلسه قبل، روش تحليل منطقى باورها، روشهايى براى تباى تغيير

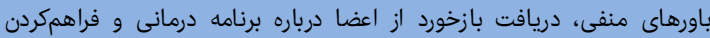

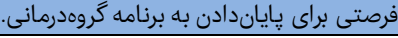

يافتهها

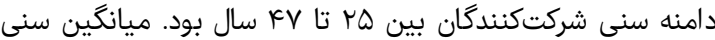

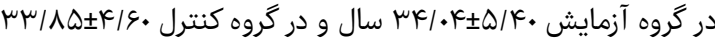

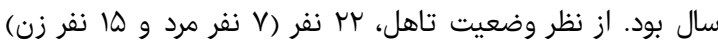

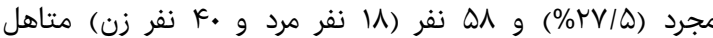

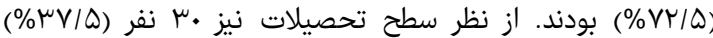

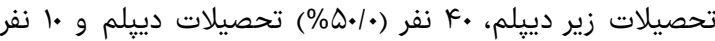

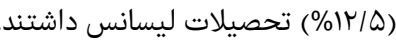

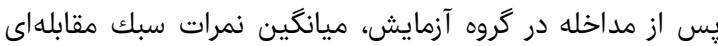

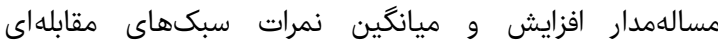

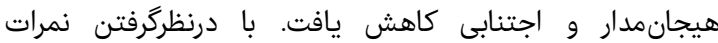

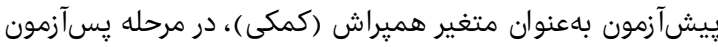

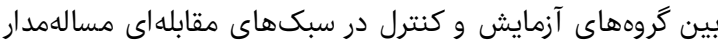

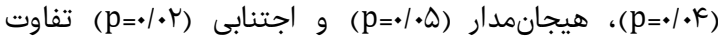

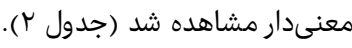

\begin{tabular}{|c|c|c|}
\hline كروه كنترل & كروه آزمايش & سبكهاى مقابلهاى \\
\hline $\begin{array}{l}F V / V V \pm r r / \varepsilon V \\
F V / \cdot I \pm r r / \& q\end{array}$ & $\begin{array}{l}r A / \Delta r \pm Y \mu / G . \\
\Delta G / K V \pm r \cdot / V V\end{array}$ & مالدمدار \\
\hline $\begin{array}{l}\Delta 1 / \Delta r \pm 1 \Lambda / 9 . \\
\Delta \cdot / 9 \cdot \pm I V / V \Delta\end{array}$ & $\begin{array}{l}\Delta 1 / \& \Delta \pm 19 / k r \\
k r / V \cdot \pm 19 / \Lambda F\end{array}$ & \\
\hline 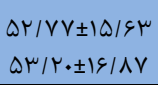 & $\begin{array}{l}\Delta r / / \Delta \pm \backslash \Lambda / Q \varphi \\
K r / I V \pm r r / \Delta \Lambda\end{array}$ & \\
\hline
\end{tabular}

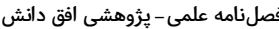




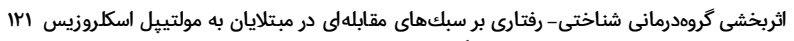

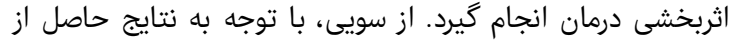

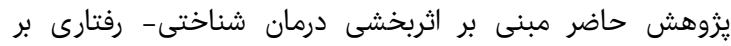

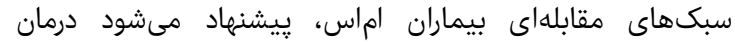

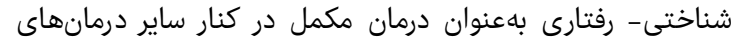

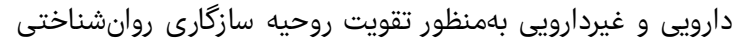
مورد توجه قرار گيرد.

نتيجه مداخله مبتنى بر گروهدرمانى شناختى- رفتارى منجر بـ به افزايش

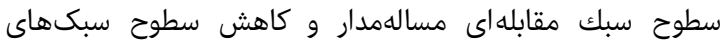

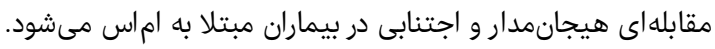

تشكر و قدردانى: از تمامى بيماران و كسانى كه در اجراى اين

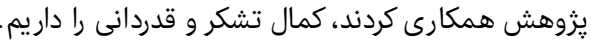

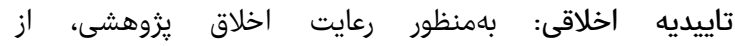

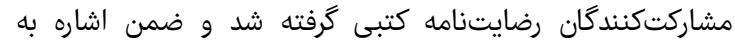

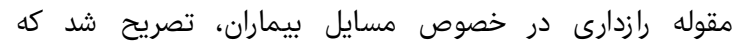

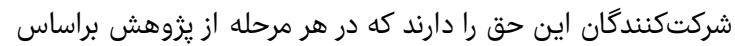

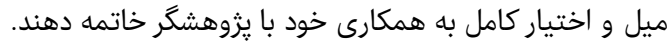

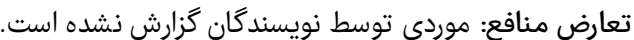

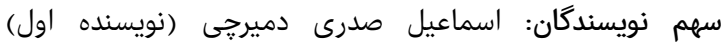

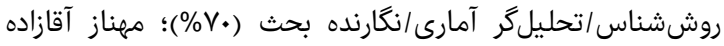

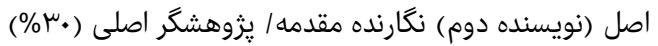

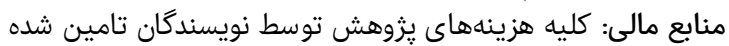

منابع

1- Khezri Moghaddam N, Ghorbani N, Bahrami Ehsan H, Rostami R. Efficacy of group therapy on reduction of psychological signs of multiple sclerosis patients. J Clin Psychol. 2012;4(1):13-22. [Persian]

2- Gold SM, Mohr DC, Huitinga I, Flachenecker P, Strenberg EM, Hessen C. The role of stress-response systems for the pathogenesis and progression of MS. Trends Immunol. 2005;26(12):644-52.

3- Malcomson KS, Dunwoody L. Lowe-Strong AS. Psychosocial interventions in people with multiple sclerosis: A review. J Neurol. 2007;254(1):1-13.

4- Mohr DC, Hart SL, Julian L, Cox D, Pelltier D. Association between stressful life events and exacerbations in multiple sclerosis: A meta-analysis. BMJ. 2004;328(7442):731.

5- Segerstrom SC, Miller GE. Psychological stress and the human immune system: A meta-analytic study of 30 years of inquiry. Psychol Bull. 2004;130(4):601-30.

6- Buljevac D, Verkooyen RP, Jacobs BC, Hop W, van der Zwaan LA, van Doorn P A, et al. Chlamydia pneumoniae and the risk for exacerbation in multiple sclerosis patients. Ann Neurol. 2003;54(6):828-31.

7- Ozbay F, Johnson DC, Dimoulas E, Morgan CA, Charney D, Southwick S. Social Support and Resilience to Stress. Psychiatry (Edgmont). 2007;4(5):35-40.

8- Dehghani A, Dehghan Nayeri N, Ebadi A. Antecedents of coping with the disease in patients with multiple sclerosis: A qualitative content analysis. Int J Community Based Nurs Midwifery. 2017;5(1):49-60.

9- Abedini SM, Montazeri S, Khalatbari J. Comparison between styles of coping with stress in patient withmultiple sclerosis and healthy people in the east of
هيجانمدار مى شود. در واقع استفاده از راهبرد هيجانمدار، فرد را از ماز براز

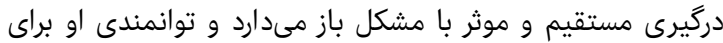

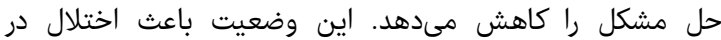

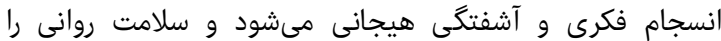

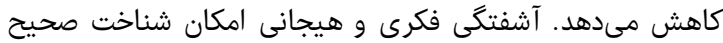

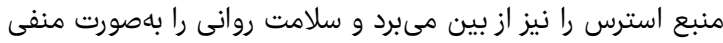

تحت تاثير قرار مىدهد [23]

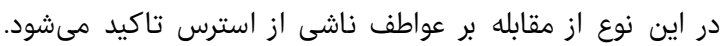

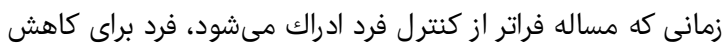

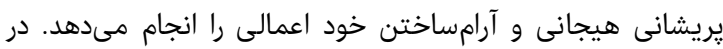

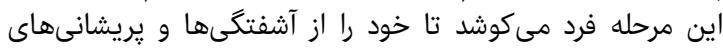

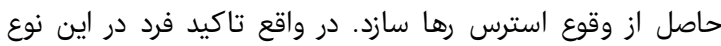

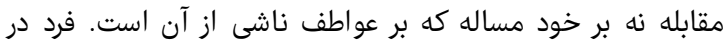

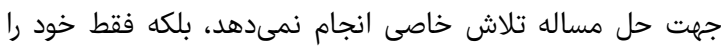

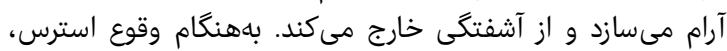

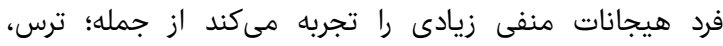

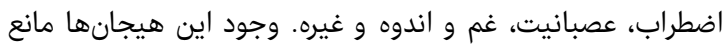

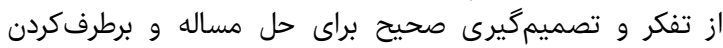

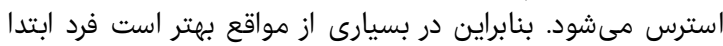

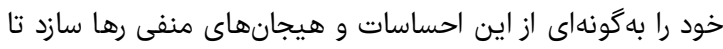

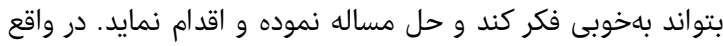

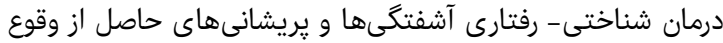

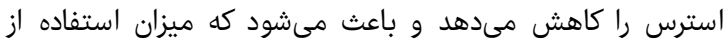

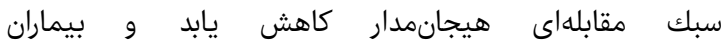
مولتيِل|سكلروزيس بر خود مساله تمركز كرده و بتوانند مشكل خود را حل كنند [16].

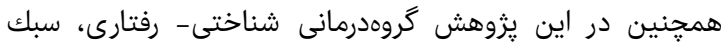

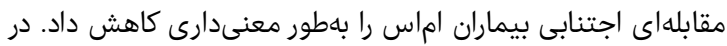

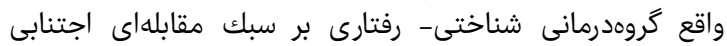

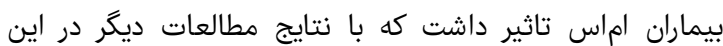

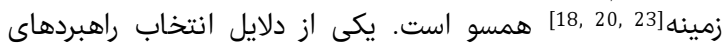

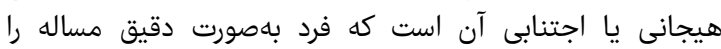

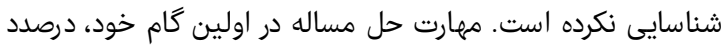

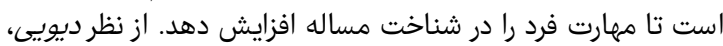

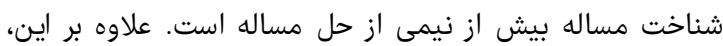

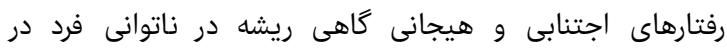

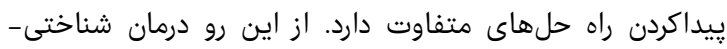

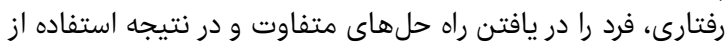

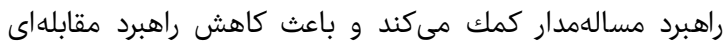

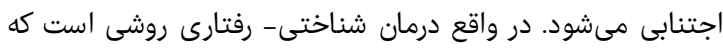

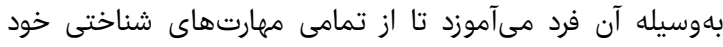

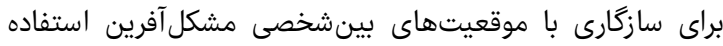

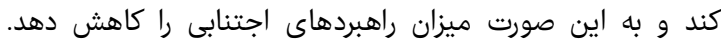

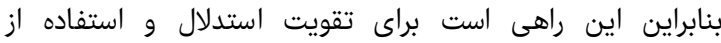

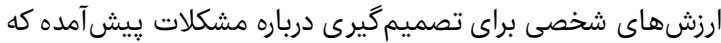

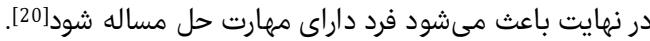

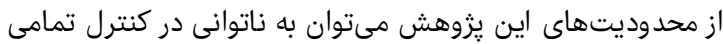

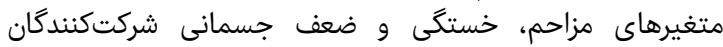

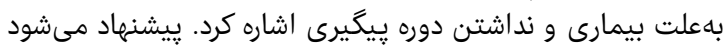

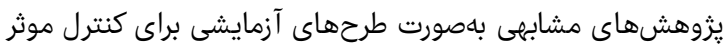

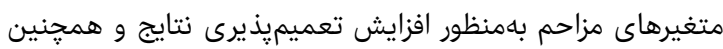

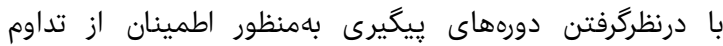


strategies in multiple sclerosis. Clin Neurol Neur surg. 2010;112(2):127-30.

17- Solari A, Radice D. Health Status of people with multiple sclerosis a community mail survey. Neurol Sci. 2001;22(4):307-15.

18- Mohamadi N, Aghaei A, Golpavar M, Etemadifar M. The effect of cognitive -behavioral group training method on the stress in patients with multiple sclerosis. Knowledge Res Appl Psychol. 2007;32(2):1-16. [Persian] 19- Thomas PW, Thomas S, Hillier C, Galvin K, Baker R. Psychological interventions for multiple sclerosis. Cochrane Database Syst Rev. 2006;(1):CD004431.

20- Nada MA, El-Mawella SM, Bayoumy HA, El-Sirafy MN. Personality trait and coping strategies in multiple sclerosis: Neuropsychological and radiological correlation. Egypt J Neurol Psychiat Neurosurg. 2011;48(1):71-8.

21- Miot HA. Sample size in clinical and experimental trials. J Vasc Bras. 2011;10(4):275-8.

22- Sharif F, Masoudi M, Ghanizadeh A, Dabbaghmanesh MH, Ghaem H, Masoumi S. The effect of cognitivebehavioral group therapy on depressive symptoms in people with type 2 diabetes: A randomized controlled clinical trial.IranJNurs MidwiferyRes.2014;19(5):529-36. 23- Aikens J E, Fischer JS, Namey M, Rudick RA. A replicated prospective investigation of life stress, coping, and depressive symptoms in multiple sclerosis. J Behav Med. 1997;20(5):433-45.
Mazandaran J Mazandaran Univ Med Sci. 2012;22(94):71-7. [Persian]

10- Bassak-Nejad S, Rahimi H, Zargar Y, Majdinasab N. Effects of stress management training based on cognitive-behavioral therapy on chronic fatigue and coping strategies in multiple sclerosis patients. Mod Care J. 2014;10(3):165-72. [Persian]

11- Mikula P, Nagyova I, Krokavcova M, Vitkova M, Rosenberger J, Szilasiova J, et al. Coping and its importance for quality of life in patients with multiple sclerosis. Disabil Rehabil. 2014;36(9):732-6.

12- Dibrivnaia KA, Enikolopova EV, Zubkova Iu V. Boľko AN. Characteristics of coping strategies in patients with multiple sclerosis (review). Zh Nevrol Psikhiatr Im S S Korsakova. 2013;113(2 Pt 2):48-52. [Russian]

13- Ghazanfari F. The relationship between mental health and coping strategies in citizenship of Khoramabad City. J Fundam Ment Health. 2008;10(37):47-54. [Persian]

14- Endler ND, Parker JD. Multidimensional assessment of coping: A critical evaluation. J Pers Soc Psychol. 1990;58(5):844-54.

15- Tartaglia MC, Narayanan S, Francis SJ, Santos AC, De Stefano N, Lapierre Y, et al. The relationship between diffuse axonal damage and fatigue in multiple sclerosis. Arch Neurol. 2004;61(2):201-7.

16- Goretti B, Portaccio E, Zippoli V, Hakiki B, Siracusa G, Sorbi $\mathrm{S}$, et al. Impact of cognitive impairment on coping 